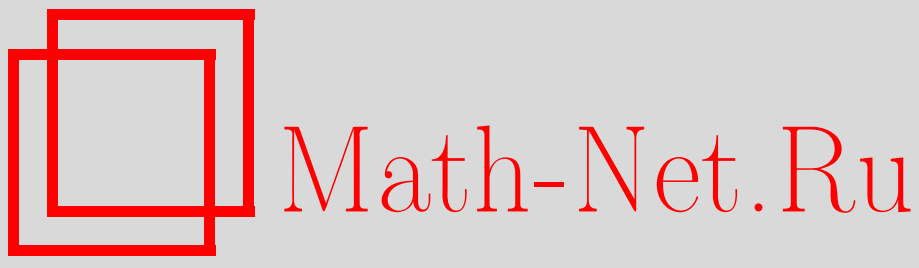

Л. С. Пасынок, Учет смешанных членов в приливном потенциале в теориях нутации неупругой земли, Вестн. Сам. гос. техн. ун-та. Сер. Физ.-мат. науки, 2004, выпуск 26, 192-193

DOI: https://doi.org/10.14498/vsgtu199

Использование Общероссийского математического портала Math-Net.Ru подразумевает, что вы прочитали и согласны с пользовательским соглашением

http://www . mathnet.ru/rus/agreement

Параметры загрузки:

IP : 54.147 .182 .235

26 апреля 2023 г., 16:26:13

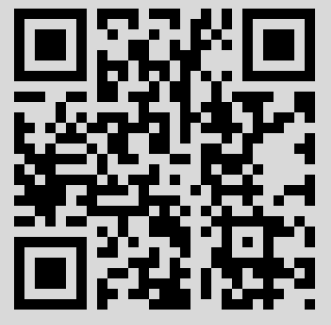




\title{
Астрометрия
}

\author{
УДК 521.1
}

\section{С.Л. Пасынок}

\section{УЧЕТ СМЕШАННЫХ ЧЛЕНОВ В ПРИЛИВНОМ ПОТЕНЦИАЛЕ В ТЕОРИЯХ НУТАЦИИ НЕУПРУГОЙ ЗЕМЛИ}

\begin{abstract}
Построено решение уравнений Эйлера для потенщиала, содержащего как периодические функции времени, так и смешанные члены Пуассона. Показано, что классическая прочедура, основанная на методе Фурье, может быть применена и в этом случае, если нутационная серия абсолютно твердой Земли была получена для потенцииала именно такого вида. Таковыми являются серии амплитуд абсолютно твердой Земли, рекомендованные в резолюииях Международного астрономического союза МАС2000. Если же используемые амплитуды нутаџии абсолютно твердой Земли получены без учета смешанных членов Пуассона, то необходимо добавлять поправки к главным нутационным гармоникам, достигающим сотен микросекунд для главных нутационных гармоник с периодом 18.6 лет.
\end{abstract}

Новейшие технологии измерений (наблюдения на РСДБ интерферометрах, лазерная локация спутников и т. д.) существенно повышают точность определения параметров вращения Земли, что выдвигает задачу пересмотра применимости стандартной процедуры вычисления нутационных углов в современных теориях нутации.

Нутационные углы входят в преобразование, описывающее переход от ЗСК (земной системы координат) к НСК (небесной системе координат). Поэтому задача построения высокоточной теории нутации является одной из важнейших задач современной астрометрии, геодезии и геодинамики. В настоящее время, благодаря использованию таких измерительных технологий, как РСДБ (радиоинтерферометрия со сверхдлинной базой), ЛЛЛ (лазерная локация Луны), ЛЛС (лазерная локация спутников), GPS (глобальная система определения местоположений), точность определения нутационных углов составляет 0.2 мс (миллисекунды) дуги. Следует отметить, что особое положение занимают измерения на РСДБ интерферометрах, поскольку именно эти наблюдения позволяют определять одновременно все параметры вращения Земли. На этом новом уровне точности необходимо проанализировать такие нелинейные эффекты, как нелинейность кинематических и динамических уравнений Эйлера, а также некоторые вопросы, касающиеся численного алгоритма вычисления этих эффектов.

Обычная процедура основана на применении метода Фурье для решения системы уравнений Эйлера [1]. Применение метода Фурье справедливо только в том случае, когда правые части являются квадратично интегрируемыми функциями времени. Однако в действительности потенциал имеет вид суммы периодических функций времени и смешанных членов Пуассона [2]:

$$
\Phi=\sum_{j}\left(\Phi_{0 j}+\Phi_{1 j} t\right) \exp \left\{i \theta_{j}(t)\right\}
$$

где $\theta_{j}(t)=\sum_{s=0}^{3} \theta_{j s} t^{s}+O\left(t^{4}\right) ; \Phi_{j s}, \theta_{j s}$ - некоторые постоянные, а время $t$ выражено в юлианских столетиях, прошедших от эпохи J2000.

Для проверки применимости классической процедуры было построено решение уравнений Эйлера, верное с той же точностью, что и разложение (1). Как известно, система уравнений Эйлера состоит из динамических и кинематических уравнений Эйлера. Их решение для потенциала (1) было найдено в виде

$$
X=\sum_{j ; k=0, \ldots, 3} X_{j k} t^{k} \exp \left\{i \theta_{j}(t)\right\}
$$

где $X$ - столбец неизвестных (отклонения экваториальной составляющей угловой скорости вращения различных частей Земли от равномерного вращения и направления наклонов их осей от направления равномерного вращения), а $X_{j s}$ - некоторые постоянные. Было проведено 
сравнение полученного решения (2) с классическим решением, основанным на методе Фурье, для теории ZР2002[3]. Согласно классической формуле [1] амплитуды неупругой Земли вычисляются по формуле

$$
\tau(\sigma)=q(\sigma) \tau_{R}(\sigma)
$$

где $q(\sigma)$ - передаточная функция, а $\widetilde{\eta}_{R}(\sigma)$ - нутационные амплитуды абсолютно твердой Земли. Задачей сравнения было решение вопроса: можно ли пользоваться формулой (3) и какие условия должны выполняться для определения нутационных амплитуд с точностью 0.1 мсек дуги? Оказалось, что формула (3) может быть применена без изменений, если $\Upsilon_{R}(\sigma)$ была получена с учетом смешанных членов Пуассона. Таковыми являются серии нутационных амплитуд абсолютно твердой Земли [4-6], рекомендованные в резолюции Международного Астрономического Союза МАС2000. Если же используемые амплитуды нутации абсолютно твердой Земли получены без учета смешанных членов Пуассона, то необходимо добавлять поправки к главным нутационным гармоникам, достигающиим нескольких сотен микросекунд для главных нутационных гармоник с периодом 18.6 лет.

Так как все современные теории нутации дают очень близкие значения для главных нутационных амплитуд [7], то этот результат справедлив не только для теории ZР2002, но и для любой другой нутационной теории, основанной на классической процедуре (3).

\section{БИБЛИОГРАФИЧЕСКИЙ СПИСОК}

1. Mathews P.M., Buffet B.A., Herring T.A., Shapiro I.I. Forced nutation of the Earth: Influence of the inner core dynamics. I. Theory. // J. Geophys. Res. ,1991. V 96. P. 8243-8257.

2. Cartwright, D. E. \& Tayler, R. J. New computation of the tide-generating potential // Geophys. J. R. Astron. Soc., 1971. V. 23. P. 45-53.

3. Zharov V.E., Pasynok S.L. Theory Of Nutation Of The Non-Rigid Earth With The Atmosphere // Astrometry from ground and from space. Abstracts book. September 25-28, 2002. Bucharest. Romania. P.

4. Bretagnon e. a. SMART97: A new solution for the rotation of the rigid Earth // Astron. Astrophys, 1998, V. 329. P. 329-338.

5. Souchay e. a. Corrections and new developments in rigid earth nutation theory. III. Final tables REN - 2000 including crossed - nutation and spin - orbit coupling effects // Astron. Astrophys. Supl.Ser., 1999. V. 135. P. 111-131.

6. Roosbeek and Dehant RDAN97: An analytical development of rigid Earth nutation series using the torque approach // Celest. Mech., 1999. V. 70. P. 215-253.

7. Pasynok S.L. IAU2000: Comparison with VLBI observations and other nutation theories // Astrometry, geodynamics and solar system dynamics: from milliarseconds to microarcseconds. Abstracts book. September 22-25, 2003. St. Petersburg. Russia. P.53.

Работа была выполнена при поддержке грантов РФФИ 01-02-16529 и 02-05-39004.

Поступила 20.12.2003 2. 